\title{
Update on acute pulmonary embolism
}

\author{
O. Sanchez*,\#, B. Planquette*,\# and G. Meyer*,\#
}

$\mathbf{T}$ he annual incidence of venous thromboembolic disease (VTE) is approximately one to two cases per 1,000 persons and is strongly age dependent, as the incidence rises to nearly $1 \%$ per year in those aged $>75$ yrs $[1,2]$. A recent epidemiological study confirmed that VTE is a major public health burden with an estimated 370,000 related deaths in 2004 in six European countries [3]. Moreover, pulmonary embolism (PE) may lead to persistent chronic disease, i.e. chronic thromboembolic pulmonary hypertension, which can be severely disabling [4-8]. Recent published data on risk factors, diagnosis, prognosis and treatment of acute PE will be the main focus of this update.

\section{RISK FACTORS}

VTE is currently considered to be the result of an interaction between patient-related and setting-related risk factors [9]. Patient-related predisposing factors are usually permanent, whereas setting-related risk factors are more often temporary (table 1). However, the predictive values of these factors are not equal and VTE can occur in patients without any identifiable predisposing factors.

\section{Pregnancy and post-partum period}

VTE is one of the leading causes of maternal morbidity and mortality. About two-thirds of VTE cases occur during pregnancy and one-third post-partum. A recent case-control study showed that the risk of VTE was increased five-fold (OR 4.6, 95\% CI 2.7-7.8) during pregnancy and increased 60-fold (OR 60.1, 95\% CI 26.5-135.9) during the first 3 months following delivery compared with nonpregnant females [10]. The risk was highest in the third trimester of pregnancy and during the first 6 weeks after delivery. The risk of thrombosis during pregnancy is affected by the presence of pro-thrombotic abnormalities. Females with Factor V Leiden or prothrombin 20210A, two common pro-thrombotic genetic variants, have a 30 to 50 -fold increased risk of VTE during pregnancy and postpartum relative to nonpregnant noncarriers [10].

\section{Cancer}

In patients with cancer, VTE is a common complication. Compared with persons without malignancy, the risk of

*Université Paris Descartes, and ${ }^{\#}$ Service de Pneumologie et Soins Intensifs, Hôpital Européen Georges Pompidou, Assistance Publique Hôpitaux de Paris, Paris, France.

CORRESPONDENCE: 0. Sanchez, Service de Pneumologie et Soins Intensifs, Hôpital Européen Georges Pompidou, 20 rue Leblanc, 75015 Paris, France. E-mail: olivier.sanchez@egp.aphp.fr

Received: June 25 2009; Accepted after revision: June 282009

PROVENANCE: Submitted article, peer reviewed. developing symptomatic VTE is seven-fold higher in patients with cancer [11]. A large population-based study determined the incidence of VTE among patients diagnosed with cancer [12]. Among 235,149 cancer cases, confirmed symptomatic VTE events were diagnosed within 2 yrs in 1.6\% [12]. In risk-adjusted models, metastatic disease at the time of diagnosis was the strongest predictor of VTE [12]. All haematological and solid tumour types have been associated with VTE but the VTE risk varies among the various types of cancer [12, 13]. BLOM et al. [11] observed the highest risk of VTE adjusted for age and sex among patients with haematological malignancies (OR 28), lung cancer (OR 22) and gastrointestinal cancer (OR 20). Adjusting for age, race and stage, diagnosis of VTE was a significant predictor of death during the first year for all cancer types [12].

In patients with symptomatic VTE, the prevalence of concomitant cancer, not known before the diagnosis of VTE and discovered by routine investigation at the time of VTE diagnosis, varies between $4 \%$ and $12 \%$ [14]. The risk of occult cancer is increased three- to four-fold in patients with idiopathic VTE compared with secondary VTE [14]. Considering the high incidence of cancer in the initial months following diagnosis VTE, screening for an underlying malignancy may be clinically relevant. The literature is, however, not concordant on whether extensive screening for occult malignancy is indicated. Recently, CARRIER et al. [15] performed a systematic review on this topic. They found that previously undiagnosed cancer is frequently found in patients with idiopathic VTE. The period prevalence of undiagnosed cancer is $6.1 \%(95 \%$ CI $5.0-7.1 \%)$ at baseline and $10.0 \%$ (95\% CI 8.6-11.3\%) from diagnosis of VTE to 12 months following diagnosis [15]. CARRIER et al. [15] demonstrated that the use of an extensive screening strategy (computed tomography of abdomen and pelvis) detected more malignancy than a limited screening strategy. However, due to the lack of detail in these studies, it is still unclear whether an increase in detection of new cancer will result in a statistical change in the detection rate of early stage or occult cancer or a decrease in cancer related morbidity and mortality.

\section{Hormone replacement therapy and oral contraceptive therapy}

Most oral contraceptives consist of a combination of oestrogen and progestogen. The reduction in dose of oestrogen over time has reduced the risk of VTE in females, although there is still a two- to five-fold increased risk [16]. Since the absolute risk of VTE is low in young females, the annual risk in users of oral contraceptives remains low at two to three cases per 10,000 [16]. The risk is highest during the first year of use. The type of progestogen affects the risk of venous thrombosis, with a twofold higher risk for contraceptives containing a third generation 


\begin{tabular}{|c|c|c|}
\hline Risk factor & Transient & Permanent \\
\hline \multicolumn{3}{|l|}{ Strong } \\
\hline Fracture, major trauma & + & \\
\hline Hip or knee replacement & + & \\
\hline Major general surgery & + & \\
\hline Spinal cord injury & + & \\
\hline \multicolumn{3}{|l|}{ Moderate } \\
\hline Active cancer & & + \\
\hline Hormone replacement therapy & + & \\
\hline Oral contraceptive therapy & + & \\
\hline Pregnancy/post-partum & + & \\
\hline Previous venous thromboembolism & + & \\
\hline Inherited thrombophilia & & + \\
\hline \multicolumn{3}{|l|}{ Weak } \\
\hline Bed rest $>3$ days & + & \\
\hline Prolonged air travel & + & \\
\hline Age & & + \\
\hline
\end{tabular}

(desogestrel and gestodene) than a second generation (levonorgestrel) progestogen [16].

Hormone replacement therapy is prescribed for the treatment of symptoms of the menopause and increases the risk of VTE by two- to four-fold [17-19]. However, in contrast to the oral route of administration, transdermal oestrogen does not have a firstpass effect through the liver and it has been suggested that this might lead to less risk of thrombosis. Recently, a large multicenter case-control study and a meta-analysis showed that oral oestrogen, but not transdermal oestrogen, increased the risk of venous thromboembolism [17, 18]. Compared with nonusers of oestrogen, the odds ratio of first diagnosis of VTE in current users of oral oestrogen was 2.5 (95\% CI 1.9-3.4) and in current users of transdermal oestrogen was 1.2 (95\% CI 0.9-1.7) [18]. The risk of VTE in females using oral oestrogen was higher in the first year of treatment (OR 4.0, 95\% CI 2.9-5.7) compared with treatment for $>1$ yr (OR 2.1, 95\% CI 1.3-3.8; $\mathrm{p}<0.05)$ [18].

\section{Inherited causes}

Deficiencies of natural coagulation inhibitors, such as antithrombin, protein $\mathrm{C}$ and protein $\mathrm{S}$, are strong risk factors for VTE but these deficiencies are rare and only account for $1 \%$ of all cases of VTE. Factor V Leiden and prothrombin (factor II) G20210A are two more common genetic variants that have been consistently found to be associated with VTE, but still only explain a small proportion of VTE cases. The search for new genetic variants associated with VTE is ongoing and is following several investigative paths. In a recent study, 19,682 single nucleotide polymorphisms (SNPs) distributed over 11,000 genes were tested in three independent datasets derived from two large population-based, case-control studies (Leiden Thrombophilia Study and MEGA study), which included $>3,000$ patients with a first diagnosis of deep vein thrombosis (DVT) and 5,000 controls [20]. Several SNPs were identified and were associated with DVT [20]. The risk-enhancing alleles for these variants were associated with a mild increase in thrombotic risk (OR varying from 1.15 to 1.39) [20].

Since genetic risk factors are either rare or have a weak overall effect, their usefulness for clinical practice is still minimal and rarely affects treatment. The search for thrombophilia is not indicated when an acquired risk factor is identified (i.e. surgery or cancer) and should be restricted in idiopathic VTE in young patients $(<50$ yrs of age) or in patients with recurrent VTE.

\section{DIAGNOSIS}

Over the past 20 yrs, many new diagnostic tests and strategies have been introduced for the diagnostic work-up of patients with suspected PE. Clinical signs, symptoms and routine tests such as chest radiography, ECG or arterial blood gases do not allow the exclusion or confirmation of PE. The available diagnostic tests also have several limitations [21]. To enforce an accurate diagnosis, diagnostic tests have now been integrated into algorithms consisting of clinical pre-test probability, Ddimer testing followed by imaging.

\section{Clinical probability}

Clinical probability assessment has become a mandatory step in the investigation of patients with clinically suspected PE. Indeed, according to Bayes' theorem, the post-test probability of PE depends not only on the intrinsic characteristics of the test used, i.e. sensitivity and specificity, but also on pre-test probability, i.e. the prevalence of $\mathrm{PE}$ in the population. Determining clinical pre-test probability of PE can be performed empirically taking into account patient's medical history and risk factors for VTE, clinical signs and routine laboratory tests. The implicit evaluation of pre-test probability was demonstrated to be relatively accurate in the PIOPED (Prospective Investigation of Pulmonary Embolism Diagnosis) study [22]. However, the empirical clinical probability is probably dependent on the physician's experience, cannot be standardised and is not reproducible. Several clinical prediction rules are available for the diagnosis of PE; two clinical prediction rules have been widely used and validated in diagnostic studies: 1) the Wells' score (table 2), and 2) the Geneva score [23, 25]. The Revised Geneva score provides a model that is independent of clinical judgement and the results of chest radiographs and arterial blood gases (table 2) [24]. The diagnostic accuracy of these rules has been shown to be comparable when retrospectively applied in one study [26]. Whichever rule is used, the prevalence of confirmed PE is $\sim 10 \%$ in the low probability category, $30 \%$ in the moderate category and $70 \%$ in the high clinical category (table 2). Recently, attempts to simplify the use of these rules for PE diagnosis have been made. Dichotomised version of the Wells' score (PE "unlikely" and "likely") have been used in two recent management studies [27, 28]. It has been proposed to simplify further the computation of these scores, attributing one point to all criteria regardless of the weight they carried in the original model $[29,30]$. These two studies have shown that this approach did not alter the diagnostic accuracy of both the Wells' and the Revised Geneva scores [29, 30].

\section{D-dimer testing}

With its high negative predictive value, the D-dimers represent an excellent noninvasive triage test in patients with suspected PE. A large variety of D-dimer assays have been evaluated and it appears that their diagnostic performances varied substantially 
TABLE 2 Clinical prediction rules for pulmonary embolism

Wells score [23]

\begin{tabular}{|c|c|c|}
\hline \multicolumn{2}{|l|}{ Variable } & Points \\
\hline \multicolumn{2}{|l|}{ Active cancer } & +1 \\
\hline \multicolumn{2}{|l|}{ Haemoptysis } & +1 \\
\hline \multicolumn{2}{|l|}{ Previous DVT or PE } & +1.5 \\
\hline \multicolumn{2}{|c|}{ Heart rate $>100$ beats $\cdot \mathrm{min}^{-1}$} & +1.5 \\
\hline \multicolumn{2}{|c|}{ Surgery or bed rest $\geqslant 3$ days within 1 month } & +1.5 \\
\hline \multicolumn{2}{|l|}{ Clinical signs of DVT } & +3 \\
\hline \multicolumn{2}{|c|}{ No alternative diagnosis as more likely than PE } & +3 \\
\hline Clinical probability & Points & PE \% \\
\hline Low & $<2$ & $2-6$ \\
\hline Intermediate & $2-6$ & $17-24$ \\
\hline High & $\geqslant 7$ & $54-78$ \\
\hline
\end{tabular}

Clinical probability (dichotomised)
Revised Geneva score [24]

\begin{tabular}{|c|c|c|}
\hline \multicolumn{2}{|l|}{ Variable } & Points \\
\hline \multicolumn{2}{|l|}{ Age $>65$ yrs } & +1 \\
\hline \multicolumn{2}{|l|}{ Active cancer } & +2 \\
\hline \multicolumn{2}{|l|}{ Haemoptysis } & +2 \\
\hline \multicolumn{2}{|l|}{ Previous DVT or PE } & +3 \\
\hline \multicolumn{2}{|c|}{ Surgery or lower limb fracture within 1 month } & +2 \\
\hline \multicolumn{2}{|c|}{ Unilateral oedema and pain at palpation } & +4 \\
\hline \multicolumn{2}{|c|}{ Spontaneously reported calf pain } & +3 \\
\hline \multicolumn{2}{|c|}{ Heart rate $75-94$ beats $\cdot \min ^{-1}$} & +3 \\
\hline \multicolumn{2}{|c|}{ Heart rate $\geqslant 95$ beats $\cdot \mathrm{min}^{-1}$} & +5 \\
\hline Clinical probability & Points & PE \% \\
\hline Low & $\leqslant 3$ & $7-12$ \\
\hline Intermediate & $4-10$ & $22-31$ \\
\hline High & $\geqslant 11$ & $58-82$ \\
\hline
\end{tabular}

DVT: deep venous thrombosis; PE: pulmonary embolism.

from one assay to another [21]. In a recent meta-analysis, DI Nisio et al. [31] calculated the diagnostic accuracy of several Ddimer tests in the diagnosis of VTE. After adjustment for study characteristics, the sensitivities of the D-dimer enzyme-linked immunofluorescence assay (Vidas; Biomérieux, Marcy L'Etolie, France; 97\%), microplate enzyme-linked immunosorbent assay (Asserachrome; Stago International, Parsippany, NJ, USA; 95\%) and latex quantitative assay (Tinaquant (Roche Diagnostics, Mannheim, Germany) and STA-liatest (Diagnostica Stago, Asnières-Sur-Seine, France); 95\%) were superior to those of the whole-blood D-dimer assay (SimpliRed; Agen Biomedical Ltd, Brisbane, Australia; 87\%), latex semiquantitative assay $(88 \%)$ and latex qualitative assay $(75 \%)$ [31]. Three large recent outcome studies using the Vidas D-dimer test showed that the 3 -month thromboembolic risk was $0 \%$ in patients with a low or intermediate clinical probability of PE left untreated on the basis of a negative test result $\left(<500 \mathrm{ng} \cdot \mathrm{mL}^{-1}\right)[28,32,33]$. In the Christopher study, the 3 -month thromboembolic risk was $0.8 \%$ in patients left untreated with the combination of PE "unlikely" (dichotomised Wells' score) and a normal D-dimer test using the Tinaquant assay $\left(<500 \mathrm{ng} \cdot \mathrm{mL}^{-1}\right)$ [28]. The whole-blood D-dimer assay SimpliRed allows ruling out PE, only in patients with low clinical probability with a 3 -month thromboembolic risk of $0.2 \%$ [34]. In patients with a high clinical probability of PE, a D-dimer test should not be performed due to theoretically limited negative predictive values of the test in patients with a high pre-test probability, i.e. prevalence of PE in this category. Moreover, it would be difficult for physicians to accept that a normal D-dimer test result over-rules their clinical judgement in patients with high clinical probability.

D-dimer tests have low specificity and low positive predictive value [31]. Indeed, D-dimers are elevated in a wide variety of conditions such as cancer, inflammation and infection. Therefore, elevated D-dimers are not useful for confirming $\mathrm{PE}$ and do not increase the clinical suspicion of PE [35].

\section{Compression ultrasonography}

Compression ultrasonography (CUS) of the lower limb veins is used as an indirect method to diagnose PE. The finding of a proximal DVT in patients suspected of PE is sufficient to warrant anticoagulant treatment without further testing [35]. The only validated diagnostic criterion for DVT is incomplete compressibility of the vein which indicates the presence of clots [35]. The specificity of CUS of proximal lower limb veins is high ( $\sim 95 \%)$. Therefore, a positive proximal CUS confirms the diagnosis of proximal DVT. The sensitivity is only $40-50 \%$ and a negative proximal CUS cannot rule out the diagnosis [35]. The diagnostic yield of CUS in patients suspected of PE might be increased by performing complete ultrasonography, including the distal veins. The proportion of patients with PE in whom DVT could be detected increased from $22 \%$ when performing proximal CUS to $43 \%$ using complete CUS, but the specificity decreased from $96 \%$ to $84 \%$ [36]. A recent study aimed at assessing whether performing complete CUS would increase the diagnostic yield of the test in 855 consecutive outpatients suspected of PE included in a multicentre, randomised 
controlled trial [37]. Patients were investigated by a sequential diagnostic strategy including clinical probability assessment, Ddimer measurement, proximal CUS and multidetector-helical computed tomography (MDCT). Proximal CUS was completed by an examination of the distal veins, the result of which was not disclosed to the physician in charge of the patient. CUS was positive in $21 \%$ of patients, of whom $10 \%$ had proximal DVT and $11 \%$ isolated distal DVT. Of the 59 patients with distal DVT, 21 (36\%) had no PE on MDCT [37]. Of those 21 patients, 20 were not given anticoagulant therapy and had an uneventful followup. Distal CUS has limited diagnostic performance for the diagnosis of PE (sensitivity 22\%, 95\% CI 17-29\%; specificity $94 \%$, 95\% CI 91-96\%) [37]. Moreover, it carries a high false-positive rate which might result in over diagnosis of $\mathrm{PE}$ and unnecessary anticoagulant treatment, impeding the use of distal CUS as a confirmatory test for PE [37].

Therefore, it appears that proximal CUS can be performed to avoid CT when CUS is positive in patients with contraindications to contrast dye and/or irradiation [35].

\section{Computed tomography}

Over the past $20 \mathrm{yrs}$, helical CT of the chest has become the procedure of choice for PE diagnosis. Diagnostic performances of helical CT have been evaluated in two types of studies: accuracy and outcome studies. Accuracy studies are designed to establish the characteristics of a diagnostic test (sensitivity and specificity) by comparing test results with a reference diagnostic test (gold standard). Outcome studies evaluate patient outcomes when a given diagnostic test or strategy is used for clinical decision-making. In the field of PE, the outcome measurement is the rate of VTE events (DVT or PE) during a 3-month follow-up period in patients left untreated by anticoagulants. The reference for comparison is the rate of DVT or PE in patients left untreated after a negative pulmonary angiography, which is $1-2 \%$, with an upper limit of the $95 \%$ confidence interval of $3 \%$ during a 3-month follow-up [38].

The reported sensitivity of single-detector helical CT ranged $53-100 \%$ and specificity ranged $81-100 \%$ in a systematic review of accuracy studies [39]. Therefore, negative singledetector helical CT alone is not sufficient for ruling out PE [21]. Several outcome studies have demonstrated that the combination of a negative single-detector helical CT and the absence of DVT on proximal lower limb CUS in patients without high clinical probability of PE left untreated by anticoagulants was associated with a 3-month VTE risk, which ranged between $1.8 \%$ (95\% CI $0.8-3.3 \%$ ) and $1.0 \%$ (95\% CI $0.5-2.1 \%)[40,41]$.

MDCT has improved the visualisation of the segmental and subsegmental pulmonary arteries. Recently, the PIOPED II study reported a sensitivity of MDCT of $83 \%$ (95\% CI 76-92\%) and a specificity of $96 \%$ (95\% CI 93-97\%) [42]. This study also demonstrated that predictive values varied substantially when clinical probability of PE was taken into account. In patients with high or intermediate clinical probability, the positive predictive value of MDCT was high ( $96 \%$ and $92 \%$, respectively) but decreased to $58 \%$ in the case of low clinical probability [42]. Negative predictive value of MDCT was high in patients with low or intermediate clinical probability $(96 \%$ and $89 \%$, respectively) but was lower in patients with high clinical probability (60\%) [42]. Several outcome studies have assessed the clinical effectiveness of MDCT [28, 32, 33]. In a study including 756 consecutive patients with clinically suspected PE, all patients with or without high clinical probability and positive D-dimer underwent both proximal CUS and MDCT [32]. Only three out of 324 patients had a positive CUS and a negative MDCT [32]. The 3-month risk of VTE in patients left untreated by anticoagulants without high-clinical probability and negative MDCT was five out of 294 (1.7\%, 95\% CI 0.7-3.9\%). The overall failure rate of the diagnostic strategy (D-dimer plus MDCT) during the 3-month follow-up was eight out of $523(1.5 \%, 95 \%$ CI $0.9-2.7 \%$ ) [32]. The Christopher study used a simplified diagnostic algorithm and included a large population of 3,306 patients with clinically suspected PE [28]. All patients categorised as "PE likely" based on the dichotomised Wells' clinical rule and those with positive D-dimer and "PE unlikely" underwent a MDCT. The 3-month risk of VTE in patients left untreated by anticoagulant on the basis of negative MDCT was $1.3 \%$ (95\% CI 0.7-2.0\%) [28]. Taken together, these two studies suggest that MDCT alone could be used without addition of CUS to exclude PE. This was definitively confirmed in a recent multicentre, randomised non-inferiority trial including 1,819 patients and comparing two strategies: D-dimer plus MDCT with and without CUS [33]. The 3-month VTE risk was $0.3 \%$ (95\% CI $0.1-1.1 \%$ ) in the D-dimer-CUS-MDCT group and $0.3 \%$ (95\% CI $0.1-1.2 \%)$ in the D-dimer-MDCT group (difference $0.0 \%, 95 \%$ CI $-0.9-0.8 \%$ ) [33]. Therefore, it appears that a negative MDCT can exclude PE in patients without high clinical probability [35]. In patients with high-clinical probability, it is further investigation is recommended by either CUS, ventilation perfusion scintigraphy or pulmonary angiography [35].

The role of CT venography remains controversial. In the PIOPED II study, the sensitivity increased from $83 \%$ for MDCT alone and to $90 \%$ for MDCT combined with CT venography with a similar specificity [42]. However, the clinical usefulness of additional CT venography is questionable since $<1 \%$ of patients clinically suspected of PE had DVT on CUS and negative MDCT [32]. Moreover, CT venography substantially increases the overall examination radiation.

\section{Diagnostic algorithms}

Various combinations of clinical probability, D-dimer, CUS, ventilation perfusion lung scintigraphy and helical CT have been evaluated. In a recent study, Roy et al. [43] aimed to assess whether physicians in routine clinical practice used evidencebased diagnostic criteria for managing patients with suspected PE. They showed that diagnostic management was inappropriate in $43 \%$ [43]. The risk for VTE during follow-up was six-fold higher in these patients than in those who received appropriate management [43]. Several independent risk factors for inappropriate management were identified: age $>75 \mathrm{yrs}$ (adjusted OR 2.27, 95\% CI 1.48-3.47), known heart failure (OR 1.53, CI 1.11-2.12), chronic lung disease (OR 1.39, CI 1.001.94), pregnancy (OR 5.92, CI 1.81-19.30), current anticoagulant treatment (OR 4.57, CI 2.51-8.31), and the lack of a written diagnostic protocol in the emergency department (OR 2.54, 95\% CI 1.51-4.28) [43]. Another retrospective cohort study including hospitalised patients with suspected PE found that inappropriate diagnostic management was associated with a nonsignificant increased risk of VTE during follow-up [44]. Recently, the European Society of Cardiology proposed 


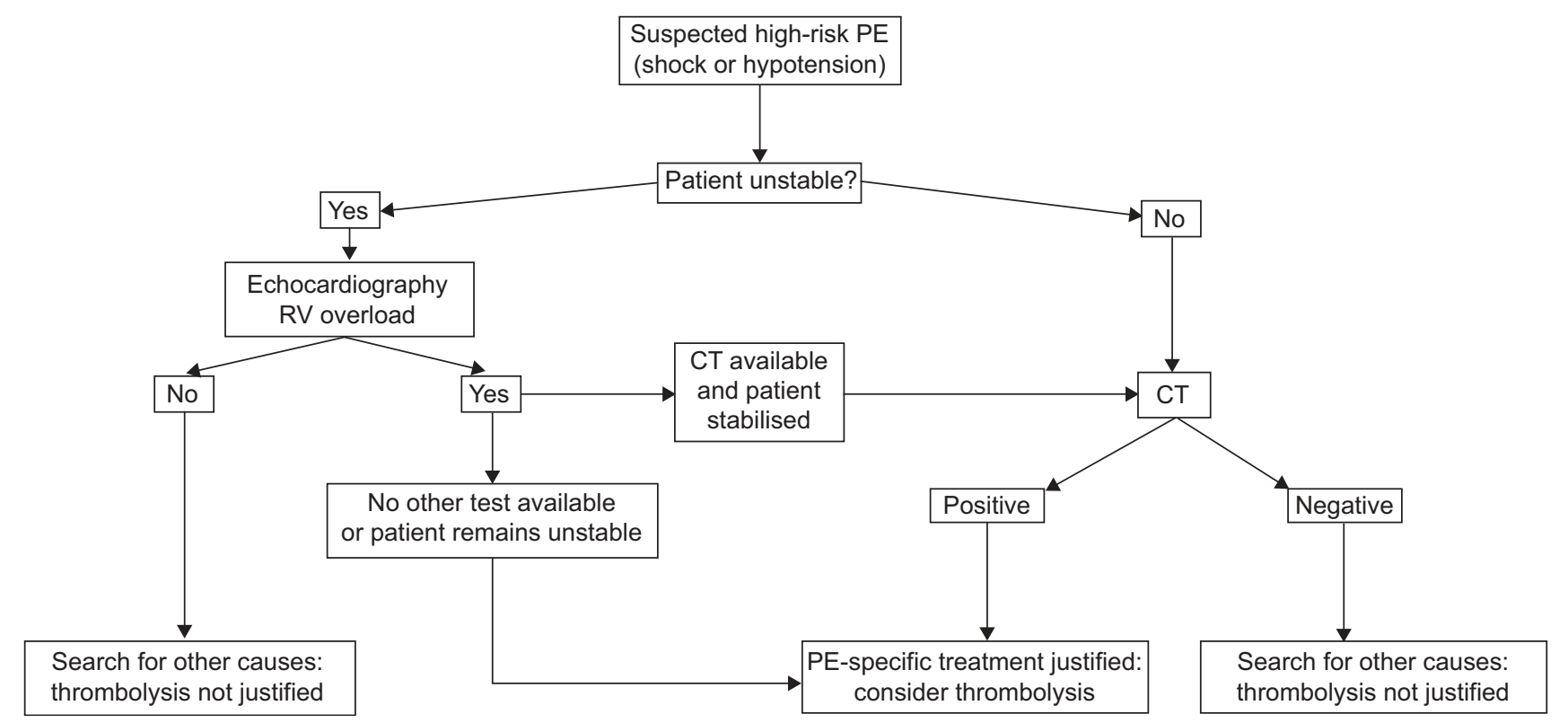

FIGURE 1. Proposed diagnostic algorithm for patients with suspected high-risk pulmonary embolism (PE), with shock or hypotension. RV: right ventricle; CT: computed tomography. Reproduced from [35] with permission from the publisher.

diagnostic algorithms for patients with suspected high-risk and non-high-risk PE (figs 1 and 2) [35].

\section{RISK STRATIFICATION}

The severity of PE should be understood as an individual estimate of risk of early (i.e. in-hospital or 30-day mortality) death rather than the degree of pulmonary vascular obstruction [35]. Therefore, PE can be stratified into several levels of early death based on the presence or the absence of risk markers [35]. Several prognostic markers have been proposed.

\section{Clinical markers}

Several years ago, data from two multicentre registries demonstrated that short-term prognosis of PE depends on haemodynamic status $[45,46]$. Definitions of haemodynamic instability varied among the studies: some studies defined it as systolic blood pressure $(\mathrm{SBP})<90 \mathrm{mmHg}$ or a reduction of $\geqslant 40 \mathrm{mmHg}$ for $\geqslant 15 \mathrm{~min}$, others defined it as SBP $<100 \mathrm{mmHg}$ and some used the shock index (cardiac frequency divided by SBP). Recently, OTERO et al. [47] compared the predictive value of these three clinical parameters on 30-day mortality in patients with acute PE included in the ongoing RIETE registry (Registro Informatizoda de Paciences con Enfermedad TromboEmbolica) [47]. On multivariate analysis, any of the three parameters were independently associated with increased 30-day mortality [47]. The shock index had a higher sensitivity $(30.5 \%$ versus $14.4 \%$ and $7.9 \%$ for SBP $<100 \mathrm{mmHg}$ and $<90 \mathrm{mmHg}$, respectively) but lower specificity $(86.3 \%$ versus $93 \%$ and $96.6 \%$ for SBP $<100 \mathrm{mmHg}$ and $<90 \mathrm{mmHg}$, respectively) [47].

Short-term prognosis of PE also depends on underlying diseases. Several clinical risk scores using other clinical variables have been proposed and validated. Using a database of patients with PE hospitalised in the USA, AUJESKY et al. [48] derived and validated a clinical prediction rule. The Pulmonary Embolism Severity Index (PESI) score includes 11 clinical variables (table 3) and divides patients into five risk classes for 30-day all-cause mortality ranging from very low to very high risk (table 4) [48]. Patients in risk classes I and II have a risk of 30-day all-cause mortality $\leqslant 2.6 \%$ and are considered low risk $[48,49]$. The PESI was recently prospectively validated using data from a randomised trial that compared two diagnostic strategies [33, 50]. In this study, the PESI classified more than half of the patients with PE as low risk (class I and class II combined) and these patients had very low overall 3-month mortality $(1.1 \%)$ [50]. These results were consistent with the original derivation and validation studies $[48,49]$. Therefore, PESI is reliable and accurately identifies patients at low risk of death.

\section{D-dimer as a risk marker}

A recently published study suggested that D-dimer could be a good predictor of mortality in patients with PE [51]. Patients who died had higher median D-dimer levels than patients who survived $\left(4,578\right.$ versus $\left.2,946 \mathrm{ng} \cdot \mathrm{mL}^{-1} ; \mathrm{p}=0.005\right)$ [51]. Mortality increased with increasing D-dimer level, rising from $1.1 \%$ in the first quartile $\left(<1,500 \mathrm{ng} \cdot \mathrm{mL}^{-1}\right)$ to $9.1 \%$ in the fourth quartile $\left(>5,500 \mathrm{ng} \cdot \mathrm{mL}^{-1}\right)$ [51]. D-dimer $<1,500 \mathrm{ng} \cdot \mathrm{mL}^{-1}$ had a sensitivity of $95 \%$ (95\% CI 74-100\%) and a negative predictive value for 30-day all-cause mortality of 99\% (95\% CI 94-100\%) [51].

\section{Markers of right ventricular dysfunction}

Death related to $\mathrm{PE}$ is mainly caused by acute right ventricular failure. Acute PE induces an abrupt increase in the right ventricular afterload, which causes dilatation and hypokinesis of the right ventricle. In the last step, the cardiac output decreases leading to reduced coronary perfusion and right ventricular ischaemia. Chronic thromboembolic pulmonary hypertension may first present as an acute episode and that some characteristics suggest a pre-existing pulmonary hypertension, such as echocardiographic evidence of right heart hypertrophy, elevated mean pulmonary artery pressure $>40 \mathrm{mmHg}$ or systolic pulmonary artery pressure $>70 \mathrm{mmHg}$ [7]. 


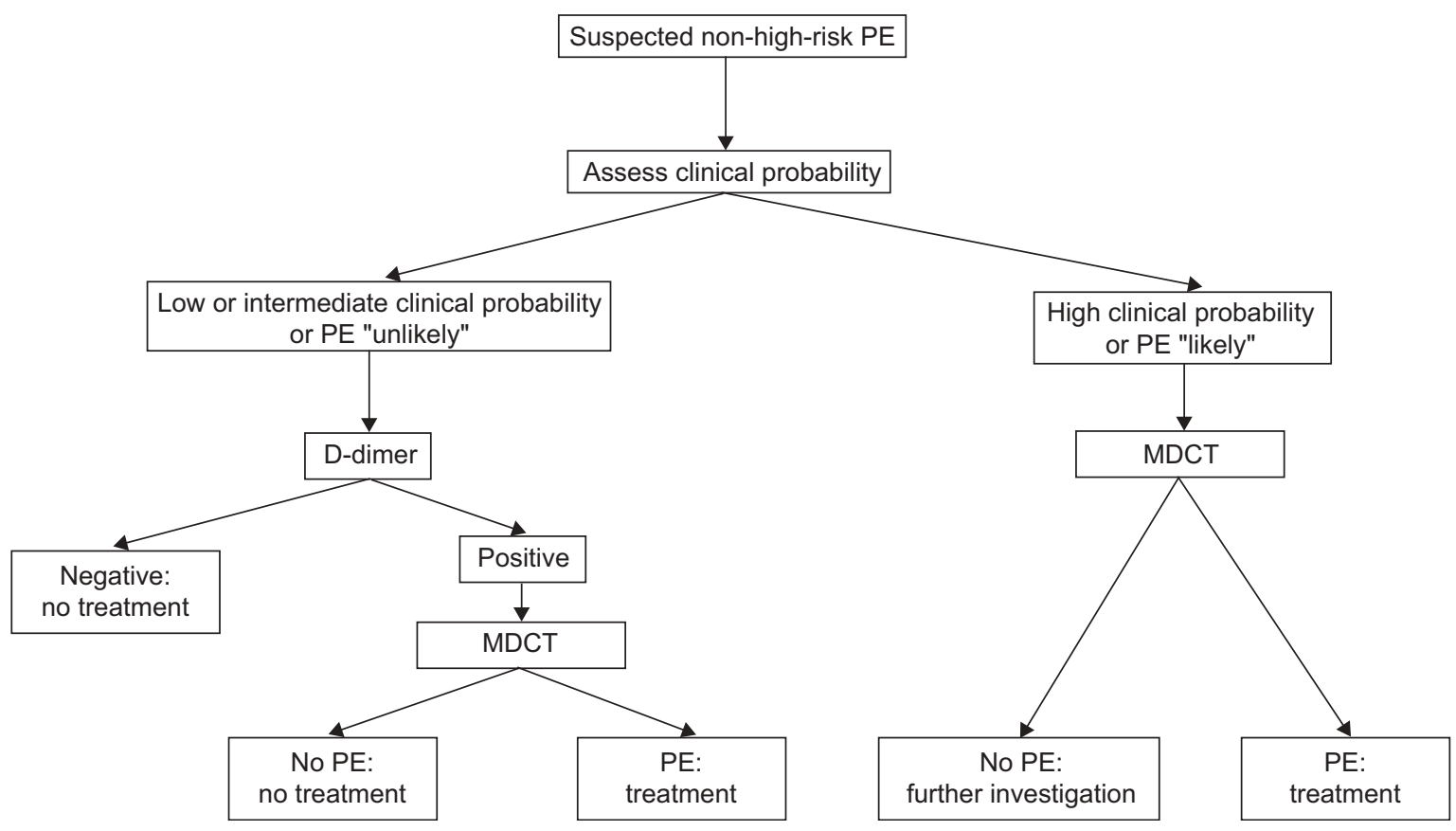

FIGURE 2. Proposed diagnostic algorithm for patients with suspected non-high-risk pulmonary embolism (PE). MDCT: multidetector-helical computed tomography Reproduced from [35] with permission from the publisher

Right ventricular dysfunction as evaluated by echocardiography is associated with high risk of mortality in patients with acute PE in several studies. A right to left ventricular enddiastolic diameter ratio $(\mathrm{RV} / \mathrm{LV}) \geqslant 0.9$ has been shown to be an independent predictor of in-hospital death (OR 2.7, 95\% CI 1.76.0) in a monocentric registry including 950 patients with acute PE who underwent echocardiography on admission [52]. This association has been confirmed in patients with normotensive $\mathrm{PE}$ in a recent meta-analysis [53]. The unadjusted risk ratio of right ventricular dysfunction as assessed by echocardiography in five studies for predicting early death was 2.5 (95\% CI 1.25.5) [53]. However, echocardiographic criteria for right ventricular dysfunction differed widely from one study to another and included right ventricular dilatation, right

\section{TABLE 3 Pulmonary Embolism Severity Index variables}

\begin{tabular}{|c|c|}
\hline Variable & Points \\
\hline Age & 1 per year \\
\hline Male sex & 10 \\
\hline Cancer & 30 \\
\hline Heart failure & 10 \\
\hline Chronic lung disease & 10 \\
\hline Heart rate $>110$ beats $\cdot \mathrm{min}^{-1}$ & 20 \\
\hline Systolic blood pressure $<100 \mathrm{mmHg}$ & 30 \\
\hline Respiratory rate $\geqslant 30$ breaths $\cdot \min ^{-1}$ & 20 \\
\hline Body temperature $<36^{\circ} \mathrm{C}$ & 20 \\
\hline Disorientation, lethargy, stupor, coma & 60 \\
\hline $\mathrm{Sa}, \mathrm{O}_{2}<\mathbf{9 0} \%$ & 20 \\
\hline
\end{tabular}

$\mathrm{Sa}, \mathrm{O}_{2}$ : arterial oxygen saturation. Reproduced from [48] with permission from the publisher ventricular hypokinesis, increased $\mathrm{RV} / \mathrm{LV}$ with different threshold and increased velocity of the jet of tricuspid regurgitation.

Recent improved CT technology (i.e. MDCT and ECG-gated helical MDCT) provides information on right ventricular dilatation only. The pooled unadjusted risk ratio of right ventricular dilatation as assessed by $\mathrm{CT}$ in two studies for predicting early death was $2.3(95 \%$ CI $0.9-6.0)$ in a recent meta-analysis [53].

New appearance of ECG signs of right ventricular strain such as inversion of $\mathrm{T}$ waves in leads $\mathrm{V} 1-\mathrm{V} 4, \mathrm{~S} 1 \mathrm{Q} 3$ pattern and incomplete or complete right bundle-branch block were found to be significant predictors of outcome [54]. These findings are useful but of limited sensitivity [54].

\section{Brain natriuretic peptides}

Brain natriuretic peptides (BNPs) and $\mathrm{N}$-terminal pro-BNP (NT-pro-BNP) are two specific markers of airway wall ventricular stress. A strong correlation between levels of BNP

\section{TABLE 4 Pulmonary Embolism Severity Index risk classes}

\begin{tabular}{lcc} 
Risk classes & Points & 30-day all-cause mortality \\
\hline Class I & $<65$ & $0 \%$ \\
Class II & $66-85$ & $1 \%$ \\
Class III & $86-105$ & $3.1 \%$ \\
Class IV & $106-125$ & $10.4 \%$ \\
Class V & $>125$ & $24.4 \%$ \\
Low risk: classes I and II & & $24.4 \%$
\end{tabular}

Reproduced from [48] with permission from the publisher. 
or NT-pro-BNP and right ventricular dysfunction has been demonstrated, as assessed by echocardiography [55]. Two recent meta-analyses demonstrated a significant relationship between high levels of BNP or NT-pro-BNP and short-term death or short-term adverse events in patients with PE [53, 55]. Patients with PE and high levels of BNP or NT-pro-BNP were at higher risk of in-hospital adverse events (OR 6.8, 95\% CI 4.410) and 30-day all-cause mortality (OR 7.6, 95\% CI 3.4-17) [55]. This association was also demonstrated in patients with normotensive PE [53].

\section{Cardiac troponins}

Cardiac troponins $\mathrm{T}$ and I have both been reported to be elevated in acute PE. The prognostic value of troponins was evaluated in several studies which have been recently pooled in three meta-analyses [53, 56, 57]. In the first meta-analysis, BECATTINI et al. [56] pooled data for normotensive and haemodynamically unstable PE and showed that elevated levels of troponin were associated with an increased risk of short-term death (OR 5.2, 95\% CI 3.3-8.4) and adverse outcome events (OR 7.0, 95\% CI 2.4-20.4). The prognostic role of troponin was also confirmed in patients with haemodynamically stable PE (OR 8.3, 95\% CI 3.6-19.3) [53]. A recent study specifically evaluated the prognostic role of troponin in 318 patients with stable PE [58]. Multivariate analysis showed that elevated troponin I was associated with increased risk of PE-related death (OR 3.7, 95\% CI 1.1-12.8) but not with 30-day all-cause death [58]. A metaanalysis including more recent studies confirmed that elevated troponin levels were associated with increased risk of all-cause mortality in patients with normotensive patients (OR 4.3, 95\% CI 2.1-8.5) [57]. However, positive and negative likelihood ratios were not extreme $(2.3,95 \%$ CI $1.7-3.1$ and 0.6 , 95\% CI $0.4-0.9$, respectively) [57]. These results indicate that elevated troponin levels do not adequately detect normotensive patients with PE at high risk from those at low risk of all-cause death [57].

\section{New markers of myocardial injury}

Heart-type fatty acid binding protein (H-FABP) is a small cytoplasmic protein abundantly expressed in tissues with an active fatty acid metabolism, such as heart and liver. Because of its small weight it appears in the circulation shortly after cell damage and demonstrates both high sensitivity and specificity in the detection of myocardial injury. Two studies investigated the prognostic value of H-FABP in patients with acute PE [59, 60]. These two studies reported that H-FABP was superior to troponin, NT-pro-BNP and myoglobin for risk stratification of PE $[59,60]$. High level of H-FABP $\left(>6 \mathrm{ng} \cdot \mathrm{mL}^{-1}\right)$ had a high negative predictive value for PE-related death or complication [60]. These promising preliminary data need to be confirmed in larger studies.

Growth differentiation factor (GDF)-15 is a distant member of the transforming growth factor- $\beta$ family. Under normal conditions, myocardium does not produce GDF-15 but its cardiac expression sharply increases after pressure overload and ischaemia. The prognostic value of GDF-15 was investigated in a study including 123 patients with acute PE [61]. On multivariate analysis, cardiogenic shock on admission, cardiac troponin I and GDF-15 were independently associated with 30day complicated outcome [61]. Further studies are needed to confirm these preliminary results.

\section{Conclusions}

Clinical assessment of haemodynamic tolerance remains the cornerstone of risk stratification of patients with acute PE. The presence of shock defined as SBP $<90 \mathrm{mmHg}$ or a drop $\geqslant 40 \mathrm{mmHg}$ for $>15 \mathrm{~min}$ defines the high-risk PE category [35]. These patients require rapid diagnosis and specific management [35]. In the remaining normotensive patients, defined as the non-high-risk PE category, the next step should be the assessment of right ventricular dysfunction or injury by imaging and/or cardiac biomarkers. However, this recommendation is supported by low level of evidence [35]. Nonhigh-risk patients without right ventricular dysfunction or injury, or with PESI class I and class II can be classified as lowrisk PE category. The presence of right ventricular dysfunction or injury seems to identify intermediate risk in normotensive patients with PE but these findings need further confirmation in large well-designed studies.

\section{TREATMENT}

Anticoagulation is the main therapy for acute PE. The main objectives of anticoagulant therapy in the initial treatment of $\mathrm{PE}$ are to prevent thrombus extension and early and late recurrences of VTE. The evidence for anticoagulant treatment is based on a number of studies performed several years ago including, principally, patients with DVT with or without symptomatic PE. Patients with PE comprise only a minority of the patients studied and their outcome is not given separately. However, patients with PE have higher risk of early death due to an initial episode or recurrence from VTE than patients with DVT. Therefore, recent randomised trials have specifically included patients with PE $[62,63]$.

\section{Initial anticoagulant treatment}

Rapid anticoagulation can only be achieved with parenteral anticoagulants, such as unfractionated heparin (UFH), low molecular weight heparin (LMWH) or fondaparinux. In the case of high-clinical probability and in the absence of contraindication, this treatment should be considered while awaiting definitive diagnostic confirmation [35, 64]. Treatment with UFH followed by warfarin is a safe and effective therapeutic option for patients with PE. However, its use is hampered by the need for an intravenous line and frequent dose adjustment and biological monitoring with activated partial thromboplastin time. The results of the recent FIDO (Fixed Dose Heparin) study suggest that this approach may be simplified [65]. In this study, 708 patients with VTE, including 134 patients with $\mathrm{PE}$, were randomised to receive subcutaneous LMWH twice daily or subcutaneous UFH at a fixed dose (initial dose of $330 \mathrm{IU} \cdot \mathrm{kg}^{-1}$ followed by $250 \mathrm{IU} \cdot \mathrm{kg}^{-1}$ b.i.d.) without monitoring and dose adjustment [65]. Recurrence and bleeding rates were low in both groups and did not differ significantly, allowing the authors to conclude that fixed-dose and unmonitored subcutaneous UFH is as effective as fixed doses of LMWH [65]. Compared with UFH, LMWHs have a longer plasma half-life and less inter-individual variability in anticoagulant response to fixed doses. As a result of their pharmacokinetic properties, a stable and sustained anticoagulant effect is achieved when LMWH is administered subcutaneously, once or twice daily, without laboratory monitoring. Several randomised controlled trials have compared fixed doses of subcutaneous LMWH to adjusted doses of 
UFH for initial treatment of DVT and PE. These results were pooled in different meta-analyses. QUINLAN et al. [66] found a nonsignificant trend in favour of LMWH for recurrent VTE (relative risk (RR) 0.68, 95\% CI 0.42-1.09), major bleeding (RR $0.67,95 \%$ CI $0.36-1.27$ ) and death (RR 0.77, 95\% CI 0.52-1.55) at 3 months [66]. There is no convincing evidence to suggest that the risk of recurrent VTE or bleeding depends on anti-factor Xa activity (anti-Xa) in patients receiving $\mathrm{LMWH}$ for the treatment of VTE. LMWH may accumulate, increasing the risk of bleeding, in patients with renal failure. Therefore, in these patients it is recommended to use dose-adjusted UFH rather than LMWH with anti-Xa measurement. UFH is also the only available option for the initial treatment of patients with PE for patients with a high risk of bleeding [64]. Fondaparinux, a synthetic pentasaccharide, was evaluated in the Matisse PE study [63]. In this study, 2,213 patients with PE were randomised to receive fondaparinux subcutaneously once daily at a fixed dose adjusted to body weight or an intravenous adjusted dose of UFH [63]. Oral anticoagulant treatment was started after 1-3 days of treatment in both groups and was continued for $\geqslant 3$ months. Subcutaneous fondaparinux was associated with a similar rate of recurrent VTE $(3.8 \%$ versus $5.0 \%$ (UFH group) at 3 months; absolute difference $-1.2 \%, 95 \%$ CI $-3.0-0.5 \%)$, major bleeding (1.2\% versus $1.1 \%$ (UFH) at the end of initial treatment) and rate of death (5.2\% versus $4.4 \%$ (UFH) at 3 months) as compared with adjusted intravenous UFH [63]. These results indicate that fondaparinux was not inferior to UFH for initial treatment of patients with PE [63]. As no proven heparin-induced thrombocytopenia has ever been observed with fondaparinux, platelet count monitoring is not needed with this treatment. Fondaparinux is contraindicated in severe renal failure (creatinine clearance $<30 \mathrm{~mL} \cdot \mathrm{min}^{-1}$ ).

Taken together, these results allow proposing recommendations on initial anticoagulant treatment for patients with PE with high level of evidence $[35,64]$. In the eighth edition of the American College of Chest Physicians guidelines, it is recommended that initial treatment of $\mathrm{PE}$ is with subcutaneous LMWH, i.v. UFH, monitored or fixed dose of subcutaneous UFH, or subcutaneous fondaparinux (all grade 1A) for $\geqslant 5$ days and until the international normalised ratio is $\geqslant 2$ for $\geqslant 24 \mathrm{~h}$ [64]. LMWH are recommended over UFH in the absence of contraindication in patients with non-high-risk PE $[35,64]$. Initiation of vitamin $K$ antagonists (VKA) is recommended on the first treatment day (grade 1A) [64].

\section{Thrombolytic treatment}

There is good evidence that thrombolytic therapy accelerates resolution of $\mathrm{PE}$ and results in more rapid haemodynamic improvement. The evidence that thrombolytic therapy improves clinical outcome remains controversial. During the past 30 yrs, several randomised trials including $<800$ patients have compared the efficacy and the safety of thrombolytic therapy plus anticoagulation versus anticoagulation alone. The results of these studies have been summarised in a recent metaanalysis, which included 11 studies totalling 748 patients with PE of varying severity [67]. Pooled data from five studies (254 patients) that included (not exclusively) patients with haemodynamically unstable PE suggested a trend toward reduction of recurrent $\mathrm{PE}$ ( $3.9 \%$ versus $7.1 \%$; OR $0.61,95 \%$ CI $0.23-1.62)$ and death $(6.2 \%$ versus $12.7 \%$; OR $0.47,95 \%$ CI $0.2-1.1)$, and an increase of major bleeding (21.9\% versus $11.9 \%$; OR $1.98,95 \%$ CI 1.00-3.92) with fibrinolysis [67]. The reduction reached statistical signification when combining recurrent PE or death $(9.4 \%$ versus 19.0\%; OR 0.45, 95\% CI 0.22-0.92) [67]. Therefore, thrombolysis should be prescribed in patients with high-risk PE unless there are absolute contraindications to its use [35, 64].

Patients with low-risk PE have a good short-term prognosis under anticoagulant treatment. The results from pooled studies that included patients with normotensive PE revealed no clinical benefit in this group [67].

To date, the largest and most recent randomised trial of thrombolytic therapy versus heparin alone included 256 patients with normotensive PE and either echocardiographic or ECG evidence of right ventricular dysfunction [68]. The primary end-point was in-hospital death or clinical deterioration requiring an escalation of treatment, which was defined as catecholamine infusion, secondary open-label thrombolysis or cardiopulmonary resuscitation. The incidence of the primary end-point was significantly higher in the heparin plus placebo group than in the heparin plus alteplase group $(p=0.006)$ [68]. Tissue plasminogen activator, compared with placebo, halved the frequency of escalation of therapy and did not increase major bleeding. However, open-label thrombolysis as rescue therapy was the main form of escalation of therapy, and as the decision to use open label rescue thrombolysis was subjective and could be made after unblinding, this component of the primary outcome has been criticised. Therefore, further studies are required to determine the risks and benefits of thrombolytic treatment in patients with normotensive $\mathrm{PE}$ and right ventricular dysfunction. A large European multicentre, double blind, placebo-controlled trial (PEITHO study) has been initiated and is currently under way. This study will randomise 1,100 patients with normotensive $\mathrm{PE}$, right ventricular enlargement on echocardiography $(\mathrm{RV} / \mathrm{LV}>0.9)$ and elevated level of cardiac troponin to receive either a bolus regimen of tenecteplase plus heparin or heparin alone. Therefore, until the results of this trial are published, the prescription of fibrinolysis in patients with intermediate-risk PE should not be generalised and should be discussed on a case by case basis $[35,64]$.

\section{Is it possible to treat some patients with PE at home?}

Randomised controlled trials have shown that the treatment of DVT with LMWH administered primarily at home is as safe as treatment with UFH in hospital. However, as suggested in a recent systematic review including a total of 928 patients with PE, outpatient treatment of PE is not based on a high level of evidence [69]. Recently, DAVIES et al. [70] aimed to assess whether patients with PE could be managed as outpatients after early discharge from the hospital using LMWH, instead of remaining as in-patients until effective oral anticoagulation was achieved. Patients were considered unsuitable for outpatient management in the case of: admission for another medical reason; additional monitoring or requirement for oxygen; likelihood of poor compliance; pregnancy; co-existing major proximal DVT; bleeding disorders; and previous VTE while on VKA [70]. Finally, 157 out of 225 patients, selected on the absence of these criteria, received outpatient anticoagulation therapy after a median length of hospitalisation of 1 day (range 1-4 days) [70]. There were no deaths, bleeding or 
recurrent VTE during the initial phase of treatment [70]. Three patients required readmission due to complication unrelated to PE. During the 3-month follow-up, three patients died unrelated to VTE event, one had a minor bleeding complication and no VTE event occurred [70]. These results suggest that outpatient management appears safe and feasible for selected low-risk patients. A randomised controlled trial is under way to compare treatment given on an outpatient basis with initial treatment administered at hospital in patients with PE selected as low risk on the basis of the PESI low-class risk (class I or II).

\section{Long-term treatment}

The aim of long-term anticoagulant treatment is to prevent fatal and nonfatal recurrence of VTE. VKAs, at a dose adjusted to maintain a target international normalised ratio of 2.5 (range 23 ), remain the treatment of choice for long-term anticoagulant therapy. The determination of optimal duration of anticoagulant treatment represents a balance between the estimated risk of recurrence after treatment discontinuation and the risk of bleeding complications under treatment. Following the cessation of anticoagulant treatment after 3-6 months, the rate of recurrent VTE appears to be higher in patients who initially had PE than in those who initially had DVT [71-73]. The recurrence rate after treatment discontinuation is lower after PE associated with reversible risk factors (surgery, trauma, immobilisation and oestrogen therapy) than after idiopathic or unprovoked PE $[74,75]$. However, the rate of fatal PE remains low after the cessation of anticoagulant treatment and does not appear to be higher than the risk of fatal bleeding during prolonged anticoagulant treatment [76, 77]. In randomised controlled trials, the rate of recurrent VTE is generally higher in patients receiving short-term treatment of 6 weeks to 6 months than in patients given more prolonged treatment $[72,73,78]$. However, in most studies, the patients were not followed after treatment cessation and the number of patients with PE included was generally small. It remains unclear whether prolonged anticoagulant treatment reduces the long-term rate of recurrent VTE or simply delays subsequent VTE events. According to the eighth edition of the American College of Chest Physicians guidelines, patients with PE secondary to a transient risk factor should be treated for 3 months and those with idiopathic PE should be treated for $\geqslant 3$ months [64]. These patients should then be evaluated for the risks versus benefits and indefinite anticoagulant therapy is recommended in patients with a first unprovoked PE and a low risk of bleeding [64]. In the case of recurrence of unprovoked $\mathrm{PE}$, indefinite treatment is recommended [64]. Long-term treatment with LMWH during at least the first 3-6 months, as compared with warfarin, has been shown to be more effective in patients with cancer $[64,79,80]$. LMWH is followed by treatment with VKAs as long as the cancer is active [64].

\section{New antithrombotic agents}

Idraparinux is a long-acting synthetic antithrombin inhibitor administered subcutaneously once per week. In the Van Gogh PE study, 2,215 patients with symptomatic PE were randomised to receive either idraparinux $\left(2.5 \mathrm{mg} \cdot \mathrm{week}^{-1}\right)$ or standard anticoagulant treatment (either UFH or LMWH followed by VKA at a dose adjusted to maintain a target international normalised ratio of 2.5) [81]. Treatment was administered for 3 or 6 months in the two groups as decided by the local investigator. The primary outcome was symptomatic, recurrent of VTE including deaths attributed to PE. The incidence of the primary outcome at 3 months was expected to be $4 \%$ for the standard treatment group and the study was designed to demonstrate the noninferiority of idraparinux with respect of the standard treatment. After 3 months of treatment, recurrent of VTE occurred in $37(3.4 \%)$ patients on idraparinux and 18 (1.6\%) patients on standard treatment (OR 2.14, 95\% CI 1.213.78) [62]. The difference in the incidence of recurrence of VTE events between the groups originated mostly during the first 2 weeks of treatment and was related to objectively recurrent events and PE-related deaths [62]. The rate of recurrent VTE in the standard treatment group was lower than expected and even lower in patients with PE than in patients with DVT receiving the same standard treatment [62]. The rates of clinically relevant bleeding at 3 months were $5.8 \%$ in the idraparinux group and $8.2 \%$ in the standard treatment group $(p=0.02)$. At 6 months, the rates were similar in both groups.

Oral direct thrombin inhibitors such as dabigatran, and oral anti-factor $\mathrm{Xa}$ inhibitors such as rivaroxaban or apixaban are currently under phase III trials.

\section{CONCLUSION}

To date, several diagnostic algorithms combining clinical probability assessment, D-dimer testing and imaging have been validated. The adherence to these algorithms appears important, as inappropriate use can increase the recurrence of VTE and PE-related death. Clinical assessment of haemodynamic tolerance remains the cornerstone of risk stratification for patients with acute PE. The presence of shock defines patients with high-risk PE. Thrombolytic treatment associated with anticoagulants is the first-line treatment in these patients. Patients with normotensive PE and without right ventricular dysfunction or injury defines a low-risk category and have good outcome under anticoagulant therapy alone. An outpatient management appears safe and feasible for selected low-risk patients. The presence of right ventricular dysfunction or injury seems to identify intermediate risk in normotensive patients with PE. Routine use of thrombolysis in these patients is not recommended but may be considered in selected patients. A large European randomised, placebo-controlled trial is currently under way to clarify this question. Whereas initial anticoagulant treatment is well codified and based on recommendations with high level of evidence, the optimal duration of long-term anticoagulant treatment remains an important clinical question in patients with idiopathic PE. Finally, new promising antithrombotic agents are currently under evaluation.

\section{STATEMENT OF INTEREST}

None declared.

\section{REFERENCES}

1 Oger E. Incidence of venous thromboembolism: a communitybased study in Western France. EPI-GETBP Study Group. Groupe d'Etude de la Thrombose de Bretagne Occidentale. Thromb Haemost 2000; 83: 657-660.

2 Silverstein MD, Heit JA, Mohr DN, et al. Trends in the incidence of deep vein thrombosis and pulmonary embolism: a 25-year population-based study. Arch Intern Med 1998; 158: 585-593. 
3 Cohen AT, Agnelli G, Anderson FA, et al. Venous thromboembolism (VTE) in Europe. The number of VTE events and associated morbidity and mortality. Thromb Haemost 2007; 98: 756-764.

4 Bonderman D, Wilkens H, Wakounig S, et al. Risk factors for chronic thromboembolic pulmonary hypertension. Eur Respir J 2009; 33: 325-331.

5 Condliffe R, Kiely DG, Gibbs JS, et al. Prognostic and aetiological factors in chronic thromboembolic pulmonary hypertension. Eur Respir J 2009; 33: 332-338.

6 Dartevelle P, Fadel E, Mussot S, et al. Chronic thromboembolic pulmonary hypertension. Eur Respir J 2004; 23: 637-648.

7 Hoeper MM, Mayer E, Simonneau G, et al. Chronic thromboembolic pulmonary hypertension. Circulation 2006; 113: 2011-2020.

8 Humbert M. Update in pulmonary hypertension 2008. Am J Respir Crit Care Med 2009; 179: 650-656.

9 Anderson FA Jr, Spencer FA. Risk factors for venous thromboembolism. Circulation 2003; 107: 19-16.

10 Pomp ER, Lenselink AM, Rosendaal FR, et al. Pregnancy, the postpartum period and prothrombotic defects: risk of venous thrombosis in the MEGA study. J Thromb Haemost 2008; 6: 632-637.

11 Blom JW, Doggen CJ, Osanto S, et al. Malignancies, prothrombotic mutations, and the risk of venous thrombosis. JAMA 2005; 293: 715-722.

12 Chew HK, Wun $\mathrm{T}$, Harvey $\mathrm{D}$, et al. Incidence of venous thromboembolism and its effect on survival among patients with common cancers. Arch Intern Med 2006; 166: 458-464.

$13 \mathrm{Ku} \mathrm{GH}$, White RH, Chew HK, et al. Venous thromboembolism in patients with acute leukemia: incidence, risk factors, and effect on survival. Blood 2009; 113: 3911-3917.

14 Otten HM, Prins MH. Venous thromboembolism and occult malignancy. Thromb Res 2001; 102: V187-V194.

15 Carrier M, Le Gal G, Wells PS, et al. Systematic review: the Trousseau syndrome revisited: should we screen extensively for cancer in patients with venous thromboembolism? Ann Intern Med 2008; 149: 323-333.

16 Vandenbroucke JP, Rosing J, Bloemenkamp KW, et al. Oral contraceptives and the risk of venous thrombosis. N Engl J Med 2001; 344: 1527-1535.

17 Canonico M, Oger E, Plu-Bureau G, et al. Hormone therapy and venous thromboembolism among postmenopausal women: impact of the route of estrogen administration and progestogens: the ESTHER study. Circulation 2007; 115: 840-845.

18 Canonico M, Plu-Bureau G, Lowe GD, et al. Hormone replacement therapy and risk of venous thromboembolism in postmenopausal women: systematic review and meta-analysis. BMJ 2008; 336: $1227-1231$.

19 Rossouw JF, Anderson GL, Prentice RL, et al. Risks and benefits of estrogen plus progestin in healthy postmenopausal women: principal results from the Women's Health initiative randomized controlled trial. JAMA 2002; 288: 321-333.

20 Bezemer ID, Bare LA, Doggen CJ, et al. Gene variants associated with deep vein thrombosis. JAMA 2008; 299: 1306-1314.

21 Roy PM, Colombet I, Durieux P, et al. Systematic review and metaanalysis of strategies for the diagnosis of suspected pulmonary embolism. BMJ 2005; 331: 259.

22 Value of the ventilation/perfusion scan in acute pulmonary embolism. Results of the prospective investigation of pulmonary embolism diagnosis (PIOPED). The PIOPED Investigators. JAMA 1990; 263: 2753-2759.

23 Wells PS, Anderson DR, Rodger M, et al. Derivation of a simple clinical model to categorize patients probability of pulmonary embolism: increasing the models utility with the SimpliRED Ddimer. Thromb Haemost 2000; 83: 416-420.

24 Le Gal G, Righini M, Roy PM, et al. Prediction of pulmonary embolism in the emergency department: the revised Geneva score. Ann Intern Med 2006; 144: 165-171.
25 Wicki J, Perneger TV, Junod AF, et al. Assessing clinical probability of pulmonary embolism in the emergency ward: a simple score. Arch Intern Med 2001; 161: 92-97.

26 Klok FA, Kruisman E, Spaan J, et al. Comparison of the revised Geneva score with the Wells rule for assessing clinical probability of pulmonary embolism. J Thromb Haemost 2008; 6: 40-44.

27 Anderson DR, Kahn SR, Rodger MA, et al. Computed tomographic pulmonary angiography vs ventilation-perfusion lung scanning in patients with suspected pulmonary embolism: a randomized controlled trial. JAMA 2007; 298: 2743-2753.

28 van Belle A, Buller HR, Huisman MV, et al. Effectiveness of managing suspected pulmonary embolism using an algorithm combining clinical probability, D-dimer testing, and computed tomography. JAMA 2006; 295: 172-179.

29 Gibson NS, Sohne M, Kruip MJ, et al. Further validation and simplification of the Wells clinical decision rule in pulmonary embolism. Thromb Haemost 2008; 99: 229-234.

30 Klok FA, Mos IC, Nijkeuter M, et al. Simplification of the revised Geneva score for assessing clinical probability of pulmonary embolism. Arch Intern Med 2008; 168: 2131-2136.

31 Di Nisio M, Squizzato A, Rutjes AW, et al. Diagnostic accuracy of D-dimer test for exclusion of venous thromboembolism: a systematic review. J Thromb Haemost 2007; 5: 296-304.

32 Perrier A, Roy PM, Sanchez O, et al. Multidetector-row computed tomography in suspected pulmonary embolism. N Engl J Med 2005; 352: 1760-1768.

33 Righini M, Le Gal G, Aujesky D, et al. Diagnosis of pulmonary embolism by multidetector CT alone or combined with venous ultrasonography of the leg: a randomised non-inferiority trial. Lancet 2008; 371: 1343-1352.

34 Wells PS, Anderson DR, Rodger M, et al. Exculding pulmonary embolism at the bedside without diagnostic imaging: management of patients with suspected pulmonary embolism presenting to the emergency department by using a simple clinical model and Ddimer. Ann Intern Med 2001; 135: 98-107.

35 Torbicki A, Perrier A, Konstantinides S, et al. Guidelines on the diagnosis and management of acute pulmonary embolism: the Task Force for the Diagnosis and Management of Acute Pulmonary Embolism of the European Society of Cardiology (ESC). Eur Heart J 2008; 29: 2276-2315.

36 Elias A, Colombier D, Victor G, et al. Diagnostic performance of complete lower limb venous ultrasound in patients with clinically suspected acute pulmonary embolism. Thromb Haemost 2004; 91: 187-195.

37 Righini M, Le Gal G, Aujesky D, et al. Complete venous ultrasound in outpatients with suspected pulmonary embolism. J Thromb Haemost 2009; 7: 406-412.

38 van Beek EJ, Brouwerst EM, Song B, et al. Clinical validity of a normal pulmonary angiogram in patients with suspected pulmonary embolism: a critical review. Clin Radiol 2001; 56: 838-842.

39 Rathbun SW, Raskob GE, Whitsett TL. Sensitivity and specificity of helical computed tomography in the diagnosis of pulmonary embolism: a systematic review. Ann Intern Med 2000; 132: 227-232.

40 Musset D, Parent F, Meyer G, et al. Diagnostic strategy for patients with suspected pulmonary embolism: a prospective multicentre outcome study. Lancet 2002; 360: 1914-1920.

41 Perrier A, Roy PM, Aujesky D, et al. Diagnosing pulmonary embolism in outpatients with clinical assessment, D-dimer measurement, venous ultrasound, and helical computed tomography: a multicenter management study. Am J Med 2004; 116: 291-299.

42 Stein PD, Fowler SE, Goodman LR, et al. Multidetector computed tomography for acute pulmonary embolism. N Engl J Med 2006; 354: 2317-2327.

43 Roy PM, Meyer G, Vielle B, et al. Appropriateness of diagnostic management and outcomes of suspected pulmonary embolism. Ann Intern Med 2006; 144: 157-164. 
44 Jouveshomme S, Bohn I, Cazaban A. Diagnosis of pulmonary embolism in hospitalised patients: retrospective survey of an institutional standard. Eur Respir J 2007; 30: 1117-1123.

45 Goldhaber SZ, Visani L, De Rosa M. Acute pulmonary embolism: clinical outcomes in the International Cooperative Pulmonary Embolism Registry (ICOPER). Lancet 1999; 353: 1386-1389.

46 Kasper W, Konstantinides S, Geibel A, et al. Management strategies and determinants of outcome in acute major pulmonary embolism: results of a multicenter registry. J Am Coll Cardiol 1997; 30: 1165-1171.

47 Otero R, Trujillo-Santos J, Cayuela A, et al. Haemodynamically unstable pulmonary embolism in the RIETE Registry: systolic blood pressure or shock index? Eur Respir J 2007; 30: 1111-1116.

48 Aujesky D, Obrosky DS, Stone RA, et al. Derivation and validation of a prognostic model for pulmonary embolism. Am J Respir Crit Care Med 2005; 172: 1041-1046.

49 Aujesky D, Roy PM, Le Manach CP, et al. Validation of a model to predict adverse outcomes in patients with pulmonary embolism. Eur Heart J 2006; 27: 476-481.

50 Donze J, Le Gal G, Fine MJ, et al. Prospective validation of the Pulmonary Embolism Severity Index. A clinical prognostic model for pulmonary embolism. Thromb Haemost 2008; 100: 943-948.

51 Aujesky D, Roy PM, Guy M, et al. Prognostic value of D-dimer in patients with pulmonary embolism. Thromb Haemost 2006; 96: 478-482.

52 Fremont B, Pacouret G, Jacobi D, et al. Prognostic value of echocardiographic right/left ventricular end-diastolic diameter ratio in patients with acute pulmonary embolism: results from a monocenter registry of 1,416 patients. Chest 2008; 133: 358-362.

53 Sanchez O, Trinquart L, Colombet I, et al. Prognostic value of right ventricular dysfunction in patients with haemodynamically stable pulmonary embolism: a systematic review. Eur Heart J 2008; 29 : 1569-1577.

54 Geibel A, Zehender M, Kasper W, et al. Prognostic value of the ECG on admission in patients with acute major pulmonary embolism. Eur Respir J 2005; 25: 843-848.

55 Klok FA, Mos IC, Huisman MV. Brain-type natriuretic peptide levels in the prediction of adverse outcome in patients with pulmonary embolism: a systematic review and meta-analysis. $A m$ J Respir Crit Care Med 2008; 178: 425-430.

56 Becattini C, Vedovati MC, Agnelli G. Prognostic value of troponins in acute pulmonary embolism: a meta-analysis. Circulation 2007; 116: 427-433.

57 Jimenez D, Uresandi F, Otero R, et al. Troponin-based risk stratification of patients with acute nonmassive pulmonary embolism: systematic review and metaanalysis. Chest 2009; [Epub ahead of print PMID: 19465511].

58 Jimenez D, Diaz G, Molina J, et al. Troponin I and risk stratification of patients with acute nonmassive pulmonary embolism. Eur Respir J 2008; 31: 847-853.

59 Kaczynska A, Pelsers MM, Bochowicz A, et al. Plasma heart-type fatty acid binding protein is superior to troponin and myoglobin for rapid risk stratification in acute pulmonary embolism. Clin Chim Acta 2006; 371: 117-123.

60 Puls M, Dellas C, Lankeit M, et al. Heart-type fatty acid-binding protein permits early risk stratification of pulmonary embolism. Eur Heart J 2007; 28: 224-229.

61 Lankeit M, Kempf T, Dellas C, et al. Growth differentiation factor15 for prognostic assessment of patients with acute pulmonary embolism. Am J Respir Crit Care Med 2008; 177: 1018-1025.

62 Buller HR, Cohen AT, Davidson B, et al. Idraparinux versus standard therapy for venous thromboembolic disease. $N$ Engl $J$ Med 2007; 357: 1094-1104.

63 Buller HR, Davidson BL, Decousus H, et al. Subcutaneous fondaparinux versus intravenous unfractionated heparin in the initial treatment of pulmonary embolism. N Engl J Med 2003; 349: 1695-1702.

64 Kearon C, Kahn SR, Agnelli G, et al. Antithrombotic therapy for venous thromboembolic disease: American College of Chest Physicians evidence-based clinical practice guidelines (8th edition). Chest 2008; 133: Suppl. 6, S454-S545.

65 Kearon C, Ginsberg JS, Julian JA, et al. Comparison of fixed-dose weight-adjusted unfractionated heparin and low-molecularweight heparin for acute treatment of venous thromboembolism. JAMA 2006; 296: 935-942.

66 Quinlan DJ, McQuillan A, Eikelboom JW. Low-molecular-weight heparin compared with intravenous unfractionated heparin for treatment of pulmonary embolism: a meta-analysis of randomized, controlled trials. Ann Intern Med 2004; 140: 175-183.

67 Wan S, Quinlan DJ, Agnelli G, et al. Thrombolysis compared with heparin for the initial treatment of pulmonary embolism: a metaanalysis of the randomized controlled trials. Circulation 2004; 110: 744-749.

68 Konstantinides S, Geibel A, Heusel G, et al. Heparin plus alteplase compared with heparin alone in patients with submassive pulmonary embolism. N Engl J Med 2002; 347: 1143-1150.

69 Squizzato A, Galli M, Dentali F, et al. Outpatient treatment and early discharge of symptomatic pulmonary embolism: a systematic review. Eur Respir J 2009; 33: 1148-1155.

70 Davies CW, Wimperis J, Green ES, et al. Early discharge of patients with pulmonary embolism: a two-phase observational study. Eur Respir J 2007; 30: 708-714.

71 Eichinger S, Weltermann A, Minar E, et al. Symptomatic pulmonary embolism and the risk of recurrent venous thromboembolism. Arch Intern Med 2004; 164: 92-96.

72 Kearon C, Gent M, Hirsh J, et al. A comparison of three months of anticoagulation with extended anticoagulation for a first episode of idiopathic venous thromboembolism. N Engl J Med 1999; 340: 901-907.

73 Schulman S, Rhedin AS, Lindmarker P, et al. A comparison of six weeks with six months of oral anticoagulant therapy after a first episode of venous thromboembolism. Duration of Anticoagulation Trial Study Group. N Engl J Med 1995; 332: 1661-1665.

74 Agnelli G, Prandoni P, Becattini C, et al. Extended oral anticoagulant therapy after a first episode of pulmonary embolism. Ann Intern Med 2003; 139: 19-25.

75 Baglin $\mathrm{T}$, Luddington $\mathrm{R}$, Brown $\mathrm{K}$, et al. Incidence of recurrent venous thromboembolism in relation to clinical and thrombophilic risk factors: prospective cohort study. Lancet 2003; 362: 523-526.

76 Douketis JD, Gu CS, Schulman S, et al. The risk for fatal pulmonary embolism after discontinuing anticoagulant therapy for venous thromboembolism. Ann Intern Med 2007; 147: 766-774.

77 Palareti G, Leali N, Coccheri S, et al. Bleeding complications of oral anticoagulant treatment: an inception-cohort, prospective collaborative study (ISCOAT). Italian Study on Complications of Oral Anticoagulant Therapy. Lancet 1996; 348: 423-428.

78 Buller HR, Cohen AT, Davidson B, et al. Extended prophylaxis of venous thromboembolism with idraparinux. N Engl J Med 2007; 357: 1105-1112.

79 Lee AY, Levine MN, Baker RI, et al. Low-molecular-weight heparin versus a coumarin for the prevention of recurrent venous thromboembolism in patients with cancer. N Engl J Med 2003; 349: 146-153.

80 Meyer G, Marjanovic Z, Valcke J, et al. Comparison of lowmolecular-weight heparin and warfarin for the secondary prevention of venous thromboembolism in patients with cancer: a randomized controlled study. Arch Intern Med 2002; 162: 1729-1735.

81 Buller HR, Cohen AT, Davidson B, et al. Idraparinux versus standard therapy for venous thromboembolic disease. $N$ Engl J Med 2007; 357: 1094-1104. 